Jörn Pachl

Systemtechnik des Schienenverkehrs

Bahnbetrieb planen, steuern und sichern 


\section{Jörn Pachl}

\section{Systemtechnik des Schienenverkehrs}

\section{Bahnbetrieb planen, steuern und sichern}

4., überarbeitete und erweiterte Auflage

Mit 163 Abbildungen, 13 Tabellen und Beispielen

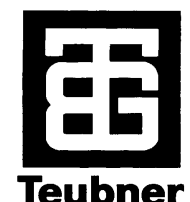

B. G. Teubner Stuttgart · Leipzig · Wiesbaden 
Bibliografische Information der Deutschen Bibliothek

Die Deutsche Bibliothek verzeichnet diese Publikation in der Deutschen Nationalbibliographie; detaillierte bibliografische Daten sind im Internet über <http://dnb.ddb.de> abrufbar.

Prof. Dr.-Ing. Jörn Pachl ist geschäftsführender Leiter des Instituts für Eisenbahnwesen und Verkehrssicherung der Technischen Universität Braunschweig. Er studierte Verkehrsingenieurwesen in Dresden und war vor seinem Ruf an die TU Braunschweig im Betriebsmanagement der Deutschen Bahn in der Region Hamburg tätig. Prof. Pachl ist Autor und Koautor mehrerer Fachbücher auf dem Gebiet des Bahnbetriebes.

1. Auflage 1999

2. Auflage 2000

3. Auflage 2002

4., überarb. und erw. Aufl. Juli 2004

Alle Rechte vorbehalten

๑ ㅇ B. G. Teubner Verlag / GWV Fachverlage GmbH, Wiesbaden 2004

Der B. G. Teubner Verlag ist ein Unternehmen von Springer Science+Business Media. www.teubner.de

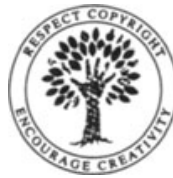

Das Werk einschließlich aller seiner Teile ist urheberrechtlich geschützt. Jede Verwertung außerhalb der engen Grenzen des Urheberrechtsgesetzes ist ohne Zustimmung des Verlags unzulässig und strafbar. Das gilt insbesondere für Vervielfältigungen, Übersetzungen, Mikroverfilmungen und die Einspeicherung und Verarbeitung in elektronischen Systemen.

Die Wiedergabe von Gebrauchsnamen, Handelsnamen, Warenbezeichnungen usw. in diesem Werk berechtigt auch ohne besondere Kennzeichnung nicht zu der Annahme, dass solche Namen im Sinne der Warenzeichen- und Markenschutz-Gesetzgebung als frei zu betrachten wären und daher von jedermann benutzt werden dürften.

Umschlaggestaltung: Ulrike Weigel, www.CorporateDesignGroup.de 


\section{Vorwort}

Die gegen Ende des 20. Jahrhunderts in vielen Ländern begonnene Reformierung und Neustrukturierung des Eisenbahnwesens führte zu einem wachsenden Bedarf an solide ausgebildeten Fach- und Führungskräften. Zur Ergänzung des bestehenden Angebots an Hochschullehrbüchern für einzelne Fachsparten der Bahntechnik fehlte längere Zeit eine fachübergreifende Beschreibung der grundsätzlichen systemtechnischen Zusammenhänge des Schienenverkehrs. Diese Lücke möchte das vorliegende Lehrbuch schließen. Das Buch wendet sich in erster Linie an Studierende technischer Studiengänge an Hoch- und Fachhochschulen, insbesondere des Verkehrswesens, des Bauingenieurwesens, der Elektrotechnik, der Automatisierungstechnik und der Informatik, die sich im Rahmen ihres Haupt- oder Vertiefungsstudiums ein Grundwissen über die Systemtechnik der Eisenbahn aneignen möchten. Es wendet sich aber ebenfalls an Teilnehmer von Trainee- und Weiterbildungsprogrammen der Eisenbahnunternehmen und der Bahnindustrie und soll auch fachlichen Quereinsteigern ermöglichen, sich mit dem System Bahn vertraut zu machen.

Im Unterschied zu Lehrwerken, die sich einer anlagenorientierten Beschreibung einzelner Komponenten der Bahntechnik widmen, besteht das Anliegen des vorliegenden Werkes in einer prozessorientierten Betrachtung des Systems Bahn mit dem Schwerpunkt der Durchführung von Zugfahrten im Eisenbahnnetz. Nach einer Einführung in die Begriffswelt des Schienenverkehrs folgt zunächst eine kurze Abhandlung der für die Systemtechnik relevanten fahrdynamischen Grundlagen. Den Hauptteil des Buches bilden die Abschnitte zur Regelung und Sicherung der Zugfolge und zur Steuerung und Sicherung der Fahrwegelemente. In diesen Abschnitten werden die maßgebenden Systemeigenschaften des Schienenverkehrs einer eingehenden Betrachtung unterzogen. Darauf unmittelbar aufbauend folgt ein Kapitel zur Leistungsuntersuchung von Eisenbahnbetriebsanlagen, in dem der Versuch unternommen wird, traditionelle Betrachtungsweisen mit neueren Erkenntnissen der Eisenbahnbetriebswissenschaft zu verbinden. Den Abschluss bilden Kapitel zu Fragen der Fahrplankonstruktion und der Betriebssteuerung.

Im Eisenbahnwesen existiert eine umfangreiche, historisch gewachsene Begriffswelt, die sich in dieser Form in anderen Sparten der Technik und des Verkehrs nicht findet. Dem mit dieser Begriffswelt nicht vertrauten fachlichen Neueinsteiger ist das Verständnis systemtechnischer Zusammenhänge mitunter erheblich erschwert. Aus diesem Grunde wurde in den Anhang des Buches ein Glossar mit Kurzdefinitionen von mehr als 200 Grundbegriffen des Eisenbahnwesens aufgenommen. 
In Inhalt und Gestaltung dieses Werkes flossen in maßgebender Weise die Erfahrungen aus der Lehrtätigkeit am Institut für Eisenbahnwesen und Verkehrssicherung der TU Braunschweig, an der Siemens Rail Automation Academy sowie aus weiteren Lehrveranstaltungen für die Aus- und Weiterbildung von Fachkräften aus Eisenbahnunternehmen und der Bahnindustrie ein.

Dem großen Interesse der Leser ist es zu danken, dass dieses Buch nach nur fünf Jahren bereits in der vierten Auflage vorliegt. In der aktuellen Auflage wurden neben einigen Aktualisierungen zur Anpassung an den Stand der Technik auch eine Reihe von Ergänzungen vorgenommen, wobei insbesondere die Anregungen aus dem Leserkreis Berücksichtigung fanden. Vorschläge für weitere Verbesserungen und Hinweise auf Fehler sind jederzeit willkommen.

Mein Dank gebührt dem Verlag und seinen Mitarbeitern für die konstruktive Zusammenarbeit, die sorgfältige Herstellung und die gute Ausstattung dieses Buches.

Braunschweig, Juni 2004

Jörn Pachl

Für Anregungen und Hinweise ist der Autor im Internet unter folgenden Adressen zu erreichen:

http://www.ifev.de/pachl

E-Mail: joern.pachl@gmx.de 


\section{Inhalt}

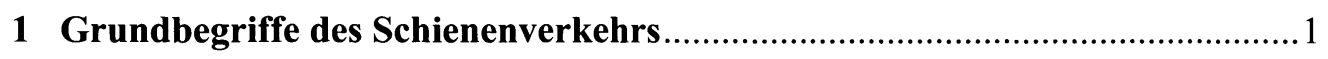

1.1 Maßgebende Systemeigenschaften …………......................................... 1

1.2 Gesetzliche Grundlagen für Bau und Betrieb von Schienenbahnen ................3

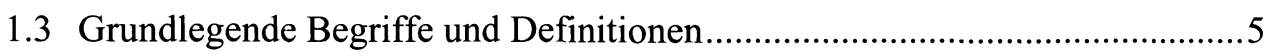

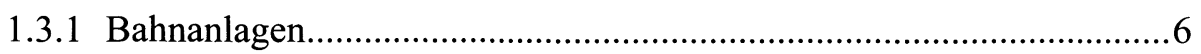

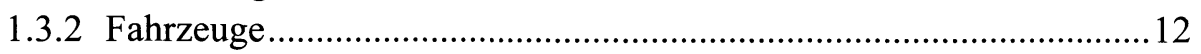

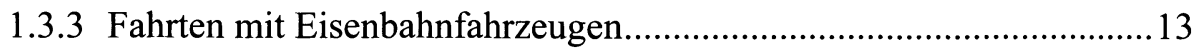

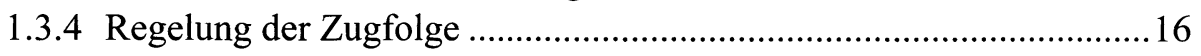

1.3.5 Abweichende Begriffswelten im Ausland ........................................18

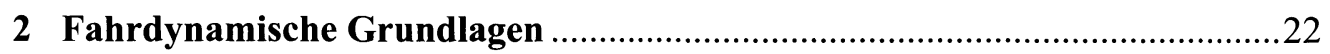

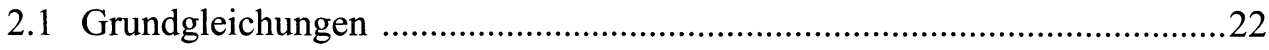

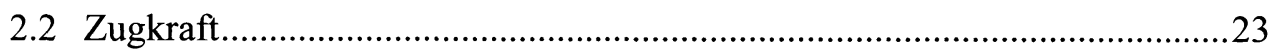

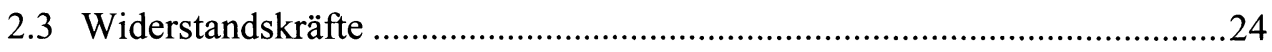

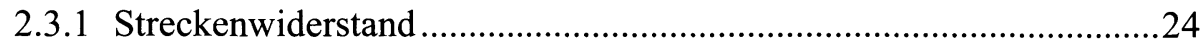

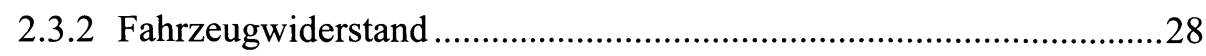

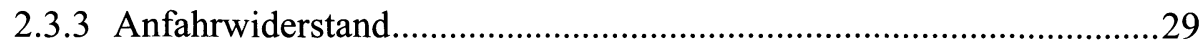

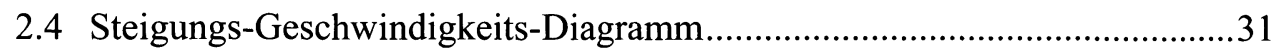

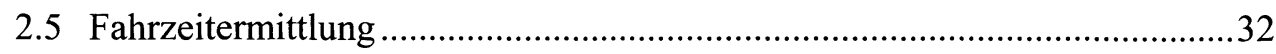

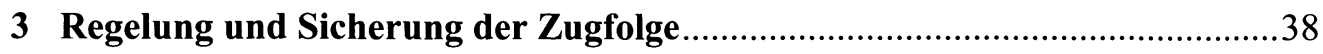

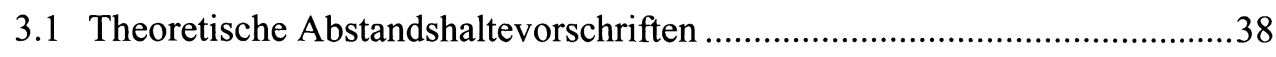

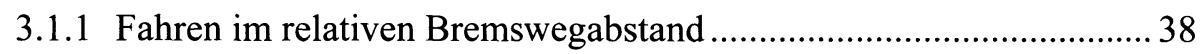

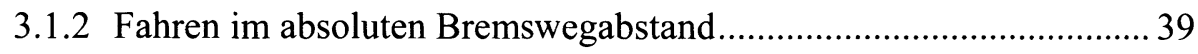

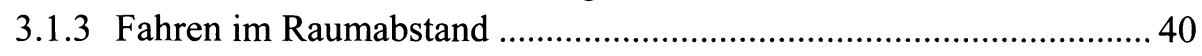


3.2 Abstandshaltetechniken im Schienenverkehr..............................................41

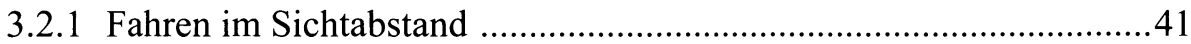

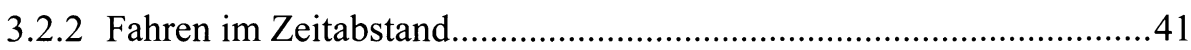

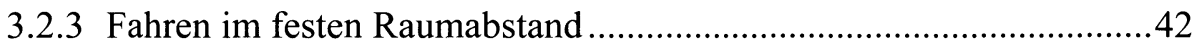

3.2.3.1 Bedingungen .....................................................................42

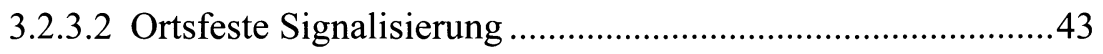

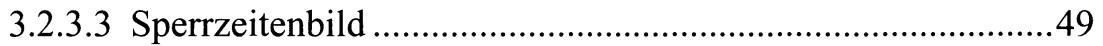

3.2.3.4 Leistungssteigerung durch Signalisierung verkürzter Blockabschnitte ....................................................53

3.3 Sicherung des Fahrens im festen Raumabstand ..........................................58

3.3.1 Verfahren ohne technische Sicherung .............................................58

3.3.1.1 Verfahren ohne technische Sicherung mit örtlicher Fahrdienstleitung .....................................................58

3.3.1.2 Verfahren ohne technische Sicherung mit zentraler Fahrdienstleitung ..................................................60

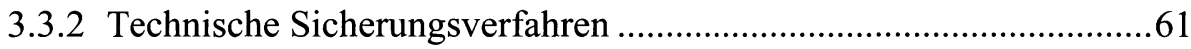

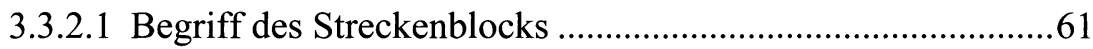

3.3.2.2 Gestaltung der Blocklogik ....................................................62

3.3.2.3 Technische Realisierung des Streckenblocks..........................66

3.3.2.4 Satellitengestützte Sicherung der Zugfolge als Alternative zum Streckenblock auf Nebenstrecken ................74

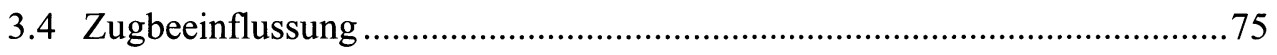

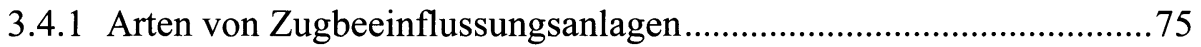

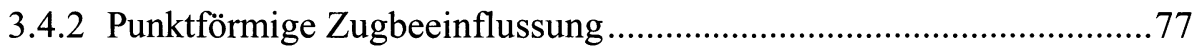

3.4.2.1 Zugbeeinflussung mit mechanischer und elektromechanischer Informationsübertragung .....................77

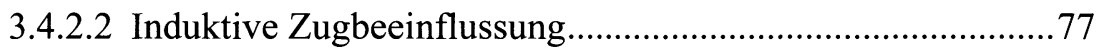

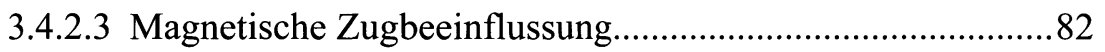

3.4.2.4 Zugbeeinflussung mit punktförmigen Datenüber-

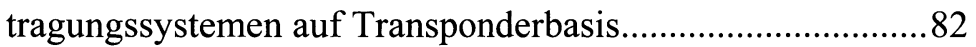

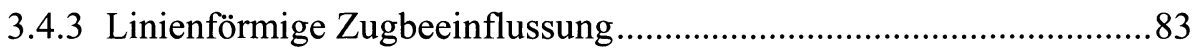

3.4.4 Vereinheitlichung der Zugbeeinflussung in Europa ............................87

3.5 Funkbasierte Verfahren zur Zugfolgesicherung ........................................90 


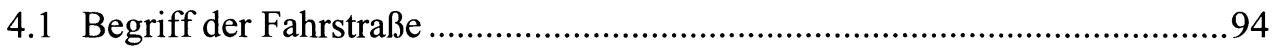

4.2 Kriterien für die Sicherung einer Fahrstraße .............................................97

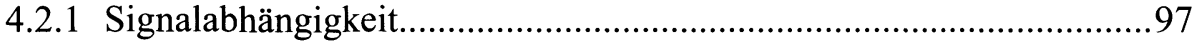

4.2.2 Fahrstraßenverschluss und Fahrstraßenfestlegung.............................99

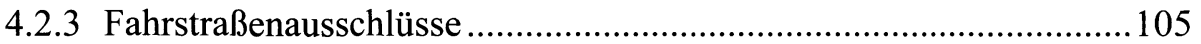

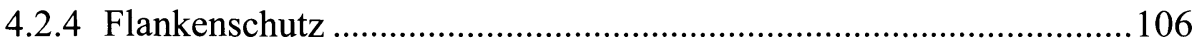

4.2.4.1 Flankenschutz gegen feindliche Zugfahrten ........................106

4.2.4.2 Flankenschutz gegen feindliche Rangierfahrten und unbeabsichtigt ablaufende Wagen .......................................107

4.2.4.3 Flankenschutz gegen das Strecken von Zügen.....................107

4.2.4.4 Erfordernis von Schutzweichen ...........................................108

4.2.4.5 Besonderheiten der Anordnung von Flankenschutz-

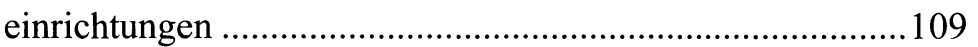

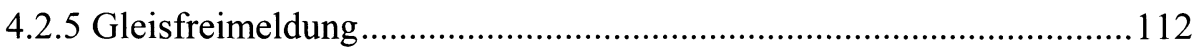

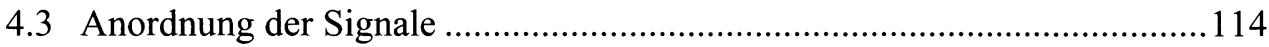

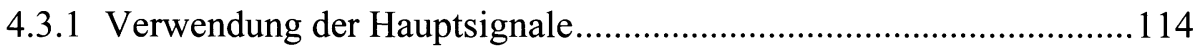

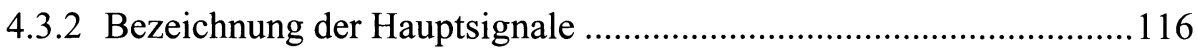

4.3.3 Abstand der Hauptsignale vom Gefahrpunkt...................................118

4.3.3.1 Maßgebender Gefahrpunkt ................................................118

4.3.3.2 Sicherung der Durchrutschwege .........................................119

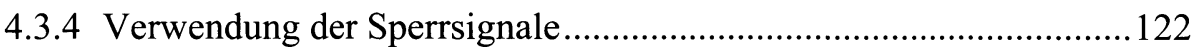

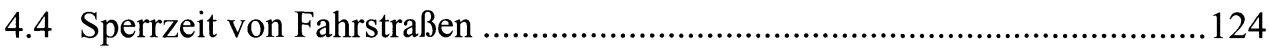

4.5 Techniken zur Fahrwegsteuerung …………..........................................127

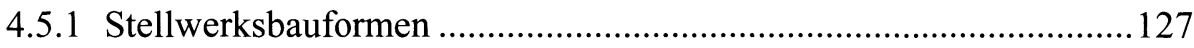

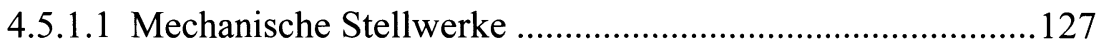

4.5.1.2 Elektromechanische und elektropneumatische

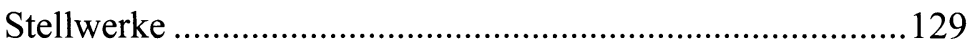

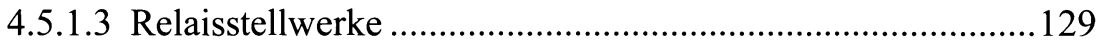

4.5.1.4 Elektronische Stellwerke (ESTW) ......................................130

4.5.2 Abbildung der Fahrstraßenlogik in Stellwerken ..............................130

4.5.2.1 Tabellarische Fahrstraßenlogik (Verschlusstabelle) .............130

4.5.2.2 Geografische Fahrstraßenlogik (Spurplanprinzip) ................132

4.5.3 Abhängigkeiten zwischen Bedienbereichen ....................................136 


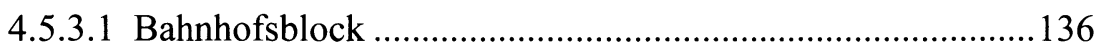

4.5.3.2 Abhängigkeiten zwischen Bedienbereichen in elektronischen Stellwerken .............................................139

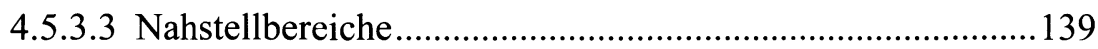

4.5.4 Streckensicherung mit den Mitteln der Fahrstraßentechnik ..............140

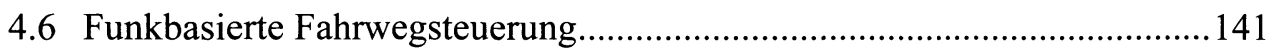

5 Leistungsuntersuchung von Eisenbahn-Betriebsanlagen............................144

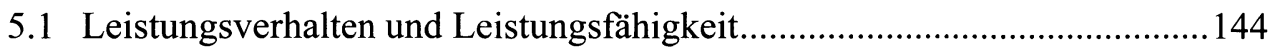

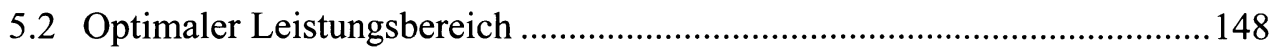

5.3 Methodik der Leistungsuntersuchungen …................................................152

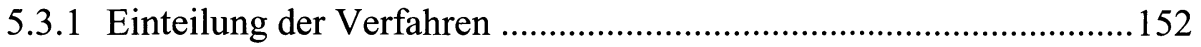

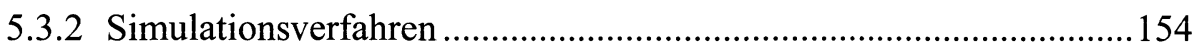

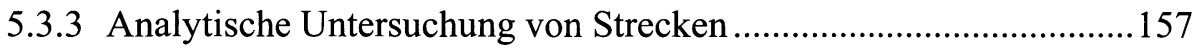

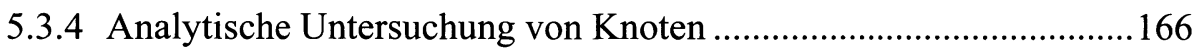

5.3.4.1 Analytische Untersuchung von Fahrstraßenknoten ..............167

5.3.4.2 Vereinfachte Verfahren zur Abbildung der betrieblichen Möglichkeiten eines Fahrstraßenknotens..................168

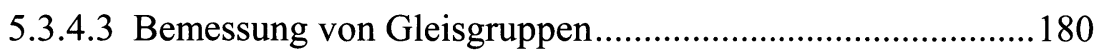

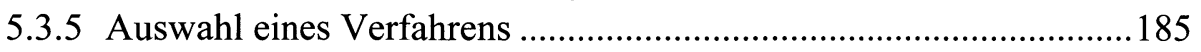

5.4 Maßnahmen zur Leistungsverbesserung.................................................185

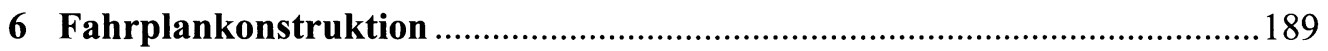

6.1 Darstellungsformen des Fahrplans für das Trassenmanagement .................190

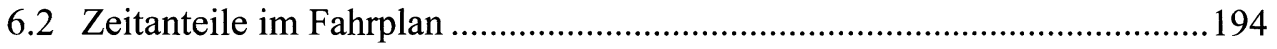

6.2.1 Bestandteile der Beförderungszeit eines Zuges .................................194

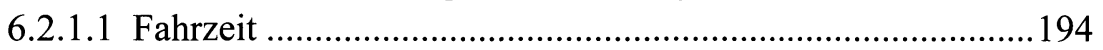

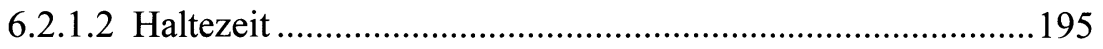

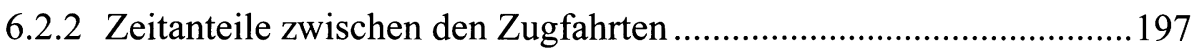

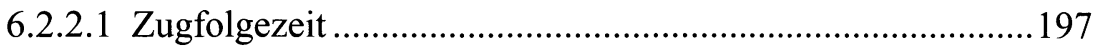

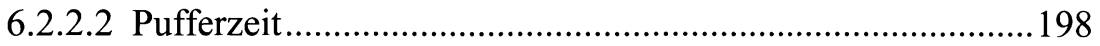

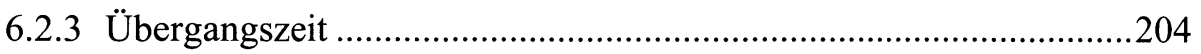

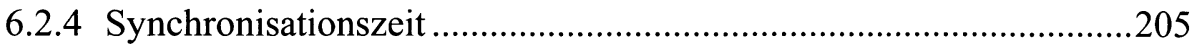




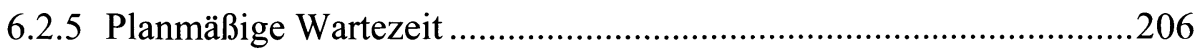

6.3 Verfahren zur Fahrplankonstruktion .......................................................209

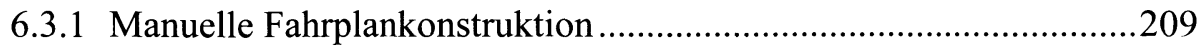

6.3.2 Rechnergestützte Fahrplankonstruktion.........................................211

6.4 Fahrplanqualität und Fahrplanleistung......................................................213

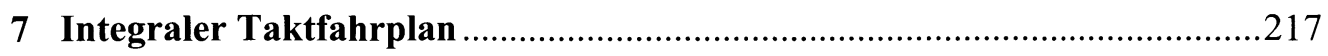

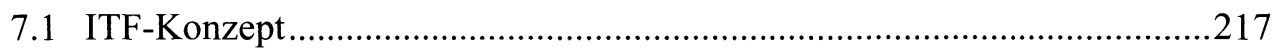

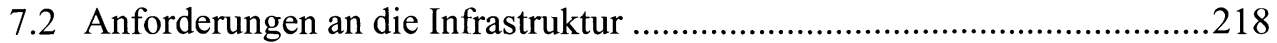

7.2.1 Strecken-Infrastruktur für feste Taktlagen ......................................221

7.2.2 Strecken-Infrastruktur für wechselnde Taktlagen..............................225

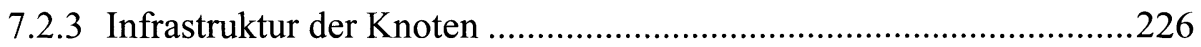

7.3 Prüfung der Fahrplanstabilität im ITF..................................................228

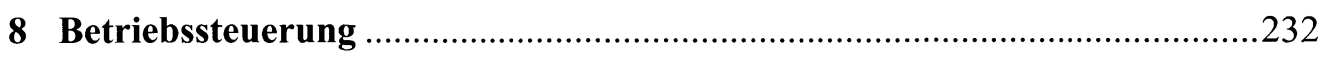

8.1 Traditionelle Organisation der Fahrdienstleitung .....................................232

8.2 Arbeitshilfen bei manueller Betriebssteuerung .........................................233

8.3 Betriebsleittechnik zur Unterstützung der Betriebsssteuerung ...................234

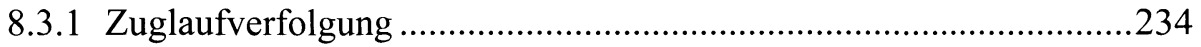

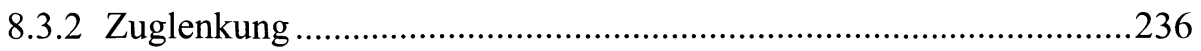

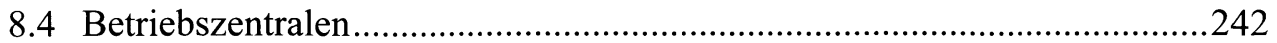

8.4.1 Aufteilung der betrieblichen Funktionalität in einer

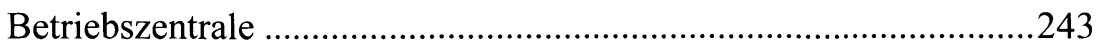

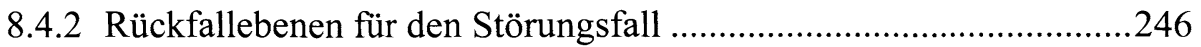

8.4.3 Grundlagen der rechnergestützten Disposition ..................................249

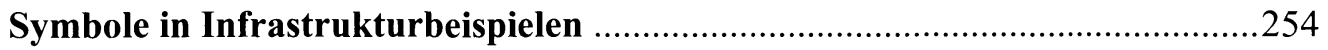

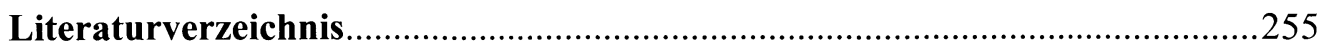

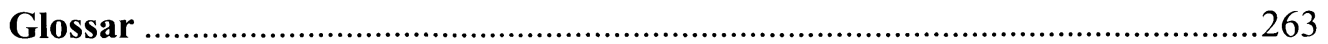

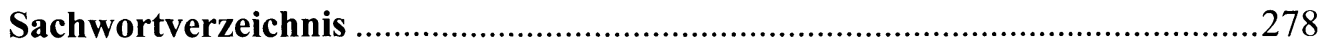

